\title{
INVESTIGACIONES
}

\section{Características y dimensión educativa en apps de educación patrimonial. Análisis a partir del método OEPE*}

\author{
Features and Educational Dimension in Heritage Education apps. \\ Analysis from the OEPE method
}

\author{
Iratxe Gillate, ${ }^{a}$ Naiara Vicent, ${ }^{a b}$ Carmen Gómez-Redondo, ${ }^{c}$ Sofía Marín-Cepeda \\ aDepartamento de Didáctica de las Ciencias Sociales, Universidad del País Vasco UPV-EHU \\ Telf.: (34) 946017544. Correo electrónico: iratxe.gillate@ehu.eus \\ bTelf.: (34) 943018364. Correo electrónico: naiara.vicent@ehu.eus \\ ${ }^{\mathrm{c}}$ Universidad de Valladolid \\ Telf.: (34) 921112200. Correo electrónico: carmen.gomez.redondo@gmail.com
}

dDepartamento de Didáctica de la Expresión Musical, Plástica y Corporal, Universidad de Valladolid Telf.: (34) 983423745. Correo electrónico: sofiavictoria.marin@uva.es

\begin{abstract}
RESUMEN
Los dispositivos móviles se han convertido en un elemento destacado de la sociedad actual. Los agentes culturales y educativos los están incorporando a sus actividades y va en aumento la oferta de apps para espacios patrimoniales. El objetivo de este artículo es conocer el estado de la cuestión en el ámbito de las apps de educación patrimonial realizadas en España. Siguiendo el método $\mathrm{OEPE}^{1}$, primeramente se ha hecho una identificación e inventariado de las apps hasta el 2016 para, posteriormente, hacer un análisis descriptivo centrándonos en su dimensión educativa. Los resultados extraídos indican cierta predisposición por parte de instancias públicas de hacer llegar este tipo de producto al usuario. La oferta existente brinda nuevas posibilidades a partir de las apps, sin embargo, no ha superado la visión tradicional de la interpretación del patrimonio, en la que el usuario mantiene una actitud pasiva sin posibilidades de crear o colaborar.
\end{abstract}

Palabras clave: aplicaciones móviles, educación, patrimonio, smartphones, evaluación.

\begin{abstract}
Mobile devices have become an important element of today's society. Cultural and educational agents are incorporating them into their offer and the offer of Apps for heritage spaces is increasing. The aim of this paper is to know the state of the issue in the field of the Heritage Education Apps made in Spain. Following the SHEO method, we have first made an identification and inventory of the Apps as far as 2016 to make, afterwards, a descriptive analysis focusing on its educational dimension. The results obtained indicate a certain willingness from public and politic authorities to close this type of product to the user. The existing offer proposes new possibilities to the use of Apps; however, it has not exceeded the traditional view of heritage interpretation, in which the user maintains a passive attitude with no possibility of creating or collaborating.
\end{abstract}

Keywords: cellular applications, education, heritage, smartphones, assessment.

Esta investigación ha sido posible gracias a la financiación recibida desde MINECO/FEDER a través de los proyectos EDU2015-65716-C2-1-R, EDU2015-65716-C2-2-R y EDU2016-78163-R, y desde el proyecto EHU 15/06.

1 Observatorio de Educación Patrimonial en España. 


\section{INTRODUCCIÓN}

El artículo que aquí se presenta es producto de la coordinación de dos proyectos de I+D vinculados al Observatorio de Educación Patrimonial en España (OEPE): "Evaluación de aprendizajes en programas de educación patrimonial centrados en los procesos de sensibilización, valorización y socialización del patrimonio cultural" y "Evaluación de programas y evaluación de aprendizajes en los ámbitos no formal e informal de la educación patrimonial".2. Ambos proyectos colaboran en la generación de un inventario de proyectos de educación patrimonial, a través del OEPE (Fontal, 2016). El mismo tiene como objetivos analizar y evaluar acciones y programas educativos, además de ser una herramienta para la investigación en el campo de la educación patrimonial (Ibáñez-Etxeberria et al., 2014; Maldonado, 2015; Marín-Cepeda et al., 2017; Sánchez, 2016).

Tanto el OEPE como ambos proyectos se sustentan sobre la idea de patrimonio inclusivo y holístico (Cuenca, 2002; Cuenca \& Estepa, 2013; Marín-Cepeda, 2014), y sobre el concepto de patrimonialización (Fontal, 2003; Mazanec et al., 2015) centrado en la dimensión identitaria o simbólico-social (Gómez-Redondo, 2013). De esta manera, el foco pasa del elemento patrimonial al sujeto patrimonializador, quien transita por la comprensión, respeto, valoración, cuidado, disfrute y, en última instancia, por la transmisión, base a su vez del conocimiento (Fontal, 2003). En definitiva, el patrimonio por sí mismo no tiene valor ni carece de él, es el ser humano quien se lo confiere y, por ello, los valores son múltiples, cambiantes y educables. Ahí es, precisamente, donde la acción educativa cobra su verdadero valor (Fontal, 2013).

De esta manera, el patrimonio ha entrado en la agenda educativa. Las propuestas de educación patrimonial ofertadas desde los espacios de educación informal (museos, sitios patrimoniales, ciudades, etc.) se han diversificado. Entre la oferta existente, en los últimos años cada vez es más común la utilización de los dispositivos móviles como soporte. Esto se debe a que estos dispositivos móviles se han convertido en un elemento cotidiano para buena parte de la sociedad (INE, 2016). Los gestores del patrimonio no se han mantenido al margen de esta realidad y la oferta de apps para este campo parece ser cada vez mayor.

Por todo ello se inicia esta investigación, que tiene por objetivo indagar en la tipología y calidad de los programas de educación patrimonial ofertados a través de apps. Para el estudio se han empleado la base de datos y el método OEPE, con el fin de realizar un análisis estadístico-descriptivo que permita aproximarnos al estado de la cuestión en España en torno a la existencia de apps vinculadas a contenidos patrimoniales.

\section{TIC, DISPOSITIVOS MÓVILES Y APPS EN EDUCACIÓN PATRIMONIAL}

Los dispositivos móviles se han convertido hoy en día en un elemento destacado de la cultura. Gracias a sus variados servicios (internet, cámara, agenda, comunicación, etc.) y su portabilidad, su valor presenta un constante auge. La clave para ello ha sido el desarrollo de internet, fuente de conocimientos, ocio y comunicación. Frente a esta revolución del mundo digital y la información, los agentes culturales y educativos han apostado por la

Ref. EDU2015-65716-C2-1-R y Ref. EDU2015-65716-C2-2-R financiados por MINECO/FEDER. A partir de este momento en el texto se hará referencia al código. 
incorporación de las tecnologías digitales. La potencialidad educativa de las mismas ha sido constatada (Kukulska-Hulme, 2007); los dispositivos móviles han sido definidos como herramientas estratégicas por la UNESCO (2013). Autores como Falcón y Torregrosa (2014) señalan que la incorporación de la tecnología a los procesos de aprendizaje puede servir para que potencialidades dormidas o desconocidas para la persona usuaria salgan a la luz.

Ante esta situación, el estudio del aprendizaje mediado por la tecnología ha captado la atención de numerosos investigadores de diversos campos, gracias a las posibilidades que ofrece para el desarrollo de procesos de aprendizaje personalizados y adaptados (Sampson \& Zervas, 2013). Esta mediación permite que el aprendiz se convierta en el centro de la experiencia educativa, ayudando a agrietar el paradigma tradicional centrado en el rol pasivo y el tratamiento homogéneo del mismo, ya que como señalan Burbules y Callister (2008) los hipertextos posibilitan un nuevo modo de presentar y organizar los contenidos, que dan lugar a cambios en las formas de conocimiento. Así, en los casos en los que existe un buen diseño, el aprendizaje mediado por la tecnología puede resultar más eficaz, puesto que parte de las diferencias y necesidades propias de cada usuario.

Dentro de este tipo de modelo de aprendizaje han ido surgiendo varias corrientes y metodologías como el mobile learning (Sharples et al., 2007) o el ubiquitous learning (Burbules, 2012). Consideramos, al igual que Brazuelo y Gallego (2011), que estas facilitan la construcción del conocimiento, la resolución de problemas de aprendizaje y el desarrollo de destrezas o habilidades diversas de forma autónoma y ubicua, gracias a la mediación de dispositivos móviles portables, a lo que añadiríamos las posibilidades que ofrece de aprendizaje en el contexto adecuado y de manera colaborativa.

Según un estudio en torno al uso de dispositivos móviles en museos (Tallon, 2013), en el 2013 un $43 \%$ de las instituciones hacía uso de ellos y un 23\% planteaba su utilización en un futuro, representando los monumentos o sitios históricos el tercer tipo de museos que hacían uso de esta tecnología. Entre los principales objetivos declarados se citan el uso de dispositivos a modo de "gancho", la incorporación de información adicional para la interpretación del patrimonio y, en tercer lugar, la intención de ofrecer un servicio más interactivo. Siendo cierto que no se utilizan todas las posibilidades que la tecnología digital ofrece, el solo hecho de poder ampliar el espacio museístico (Vavoula, 2005), la aparición de proyectos que den lugar a una interpretación contextualizada del patrimonio y la introducción de procesos de aprendizaje no lineales y asincrónicos (Falcón \& Torregrosa, 2014) pueden considerarse un gran avance para la educación patrimonial.

Las apps son aplicaciones software que se instalan en los dispositivos móviles tales como smartphones y tablets. Ofrecen un recurso específico y acceso inmediato, sin la necesidad de entrar en internet. Por lo tanto, al poder descargarse y acceder a ellas cuando y donde se desee, permiten desarrollar procesos de aprendizaje contextualizado. Según Villalonga y Marta-Lazo (2015), el éxito de una app reside en la sencillez, la usabilidad, la accesibilidad, un diseño atractivo, la adaptabilidad a las necesidades del usuario y la aplicación de los principios de la educomunicación, que defiende que los procesos comunicativos van unidos intrínsecamente a los pedagógicos. Las autoras proponen un modelo de "apprendizaje" basado en estos principios, que permitiría un viaje de aprendizajes (Falcón \& Torregrosa, 2014).

En un estado ideal, tanto el usuario como el gestor se convertirían en EMIREC (Kaplún, 1998). Es decir, con el cambio del paradigma educativo, los usuarios pasarían de ser receptores a ser EMIsores y RECeptores, o, lo que es lo mismo, se convierten en PROductores y conSUMIDORes de contenidos. Por lo tanto, en este contexto, el rol 
del educador pasa a ser el de facilitador o mediador (Osuna, 2011). Este cambio en el papel de los educadores de museos, debería darse también en las apps, donde los usuarios pasarían de ser receptores a ser EMIsores y RECeptores. Por lo tanto, la aplicación móvil adoptaría el rol de facilitador o mediador. Así pues, las apps serían herramientas de apoyo facilitadoras de experiencias educativas, pero su buen aprovechamiento dependerá de su diseño, que a su vez dependerá de los objetivos que se planteen los gestores patrimoniales.

Sin embargo, un estudio realizado por Economou y Meintani (2011), constató que la mayoría de las apps utilizadas en museos eran de carácter informativo. En el panorama español, un estudio exploratorio de López-Benito, Martínez-Gil y Santacana (2014) concluyó que entre las apps vinculadas a museos un $79 \%$ era independiente a las visitas, mientras que el $21 \%$ estaba pensado para interactuar con la colección. Por otro lado, Grevtsova (2016) analizó las apps en torno al patrimonio urbano, concluyendo que las estrategias del modelo colaborativo son poco habituales.

Por lo tanto, parece que las apps vinculadas al patrimonio siguen ejerciendo un papel similar al que ofrecían aquellas primeras propuestas que hacían las veces de audioguía o tenían un perfil marcadamente turístico (Imbert-Bouchard et al., 2013). Sin embargo, la introducción de la geolocalización, la realidad aumentada y la realidad virtual pueden aportar valores añadidos a la experiencia de los usuarios, además de la gamificación y la incorporación de las redes sociales, que posibilitan un tipo de interacción que las apps de estilo más tradicional no ofrecían.

\section{DISEÑO DE LA INVESTIGACIÓN}

Enmarcado dentro del proyecto EDU2015-65716-C2-2-R, el estudio prospectivo que aquí se presenta parte de los siguientes objetivos:

- Identificar e inventariar las diferentes apps de educación patrimonial realizadas en España hasta el año 2017.

- Conocer la dimensión educativa de las apps inventariadas.

- Conocer el estado de la cuestión en el ámbito de la educación patrimonial de las apps realizadas en España hasta el año 2017.

Para este trabajo se parte de una herramienta específica para el análisis de apps, diseñada por el grupo de investigación GIPyPAC ${ }^{3}$. Para su diseño, se realizó una revisión bibliográfica, identificando una serie de herramientas creadas para la evaluación de apps (Crescenzi \& Grané, 2016; Lee \& Cherner, 2015; Vincent, 2012) u otro tipo de entornos digitales educativos (Cabrero \& López Meneses, 2009; Guerrero, 2015), algunos de los cuales son estudios referidos al campo patrimonial (Economou \& Meitani, 2011; López Benito, 2014). Estos sirvieron de base para el diseño de la herramienta empleada en esta investigación, la cual fue sometida a discusión en un grupo de expertos (en este caso todos los miembros del grupo de investigación). Posteriormente fue validada a través de una prueba piloto (Ibáñez-Etxeberria \& Kortabitarte, 2016). Finalmente, como consecuencia de este trabajo previo y de la coordinación entre los proyectos EDU2015-65716-C2-1-R

Grupo de Investigación en Patrimonio y Paisajes Culturales (IT931-16, PPG 17/68). 
y EDU2015-65716-C2-2-R, se reelaboró la herramienta para adaptarla al método OEPE, vinculado directamente al primero de los proyecto.

El método OEPE toma como punto de partida el análisis de muestreo (Rodríguez, 2001) y la evaluación a través de instrumentos de análisis generados ad hoc en base a estándares (Stake, 2004). Metodológicamente el OEPE se describe como una secuencia de siete fases discriminatorias. Para cada fase se han generado instrumentos de análisis específicos, diseñados y validados por expertos (Fontal, 2016).

A partir de este método, se han realizado ciertas variaciones para su aplicación a esta investigación, impulsada y realizada desde el proyecto EDU2015-65716-C2-2-R. Las aportaciones de ambos proyectos que coadyuvan a esta investigación quedan reflejadas de la manera siguiente (Figura 1).

Figura 1. Combinación de aportaciones de los proyectos I+D a esta investigación

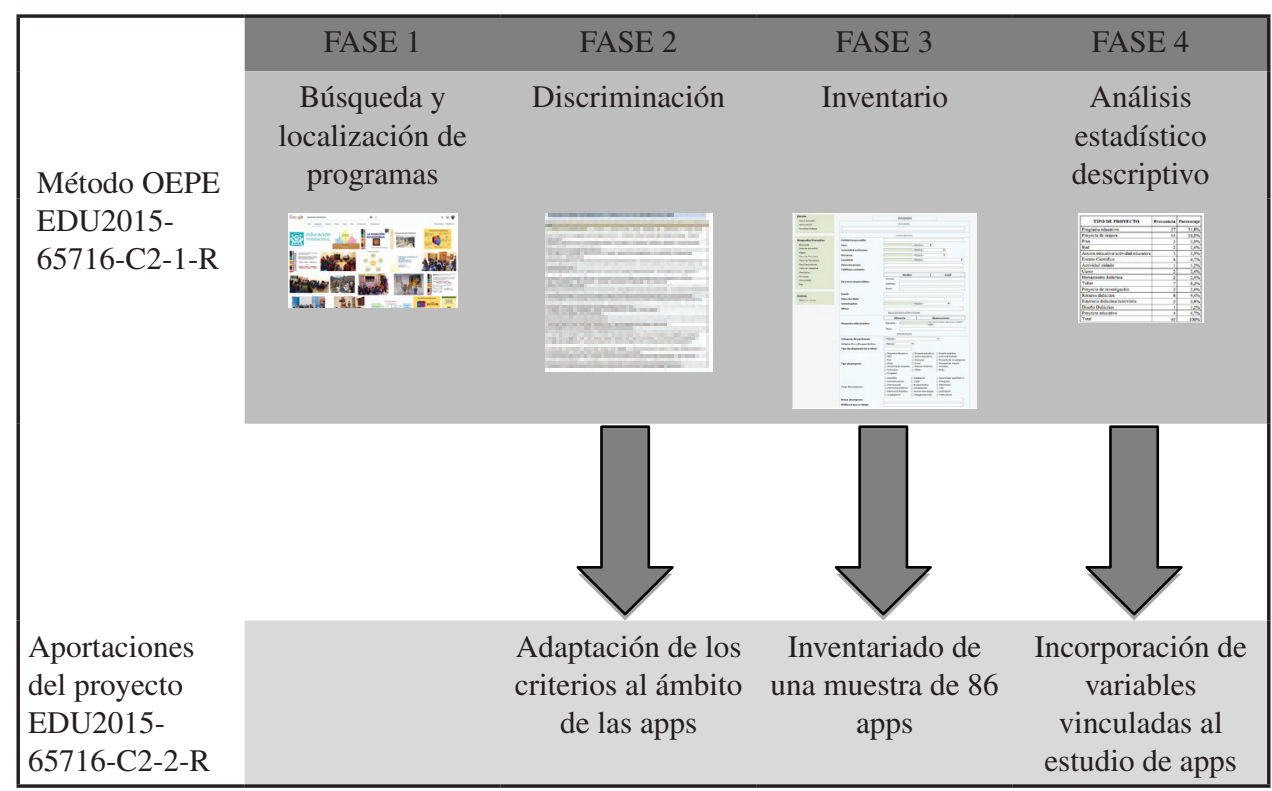

Fuente: elaboración propia.

A continuación, nos centramos en las fases específicas a las que este estudio realiza una aportación:

\section{Fase 1: Búsqueda y localización de programas}

Se realizan búsquedas en motores específicos en base a diferentes descriptores. En este caso, la búsqueda de las apps se realiza mediante el uso de palabras claves en las plataformas "store" específicas de descarga de apps para móviles tanto de Android como de Mac. El principal término de búsqueda fue "patrimonio" y sus variantes en las lenguas oficiales 
("ondarea", "patrimoni"). También se recurrió a términos más específicos que pueden relacionarse con cuestiones patrimoniales como "museos", "tradición" y "folklore". Posteriormente, se lleva a cabo una búsqueda a través de empresas promotoras en el campo de estudio, como Stoa S.L, Akting y GVAM, entre otras. Por último, se usa twitter como motor de búsqueda mediante el hashtag \#museumapp. A partir de las apps surgidas de esta búsqueda, se rastrean otras haciendo uso de la inteligencia artificial que muestra productos similares.

\section{Fase 2: Discriminación}

En esta fase comienza el filtrado de programas a través de una serie de criterios de inclusión y exclusión. Estos determinan el paso a la siguiente fase. Para la consecución de esta fase se ha adaptado la herramienta original diseñada por OEPE (Fontal, 2016), ofreciendo así unos criterios (Tabla 1) más acordes al tipo de programa de educación patrimonial que se analiza en este estudio.

\section{Fase 3: Inventario}

Se lleva a cabo un exhaustivo proceso de inventariado a través de una ficha de registro de datos que permite la sistematización de los programas y su registro en la base de datos OEPE.

En este sentido, al inventariar las apps en la base de datos OEPE se han cumplimentado los siguientes datos para cada uno de los apartados propuestos por OEPE:

- Identificación y localización: título, entidad responsable o agente que ha impulsado la app y localización geográfica de este último (país, comunidad autónoma, provincia y localidad).

- Descripción: adaptación a personas con capacidades diferentes y, en caso de que hubiese, tipo de adaptación, tipo de proyecto, breve descripción, organismos implicados y otros. Para explicitar el tipo de adaptación se ha considerado la posibilidad de cambiar el tamaño de la letra, de cambiar el tamaño de la pantalla o la existencia de voiceover. Para definir el tipo de proyecto se han empleado las categorías recogidas en la ficha de registro OEPE, todos los programas han sido inventariados como recurso didáctico y, en los que corresponda, también como itinerario didáctico. En el campo organismos implicados se ha recogido tanto el agente que impulsa el lanzamiento de la app como el desarrollador encargado de crear la aplicación. Y, por último, en la categoría otros se ha especificado la fecha de la última actualización de la app.

- Datos del diseño educativo: objetivos, orientación metodológica, estrategias de enseñanza aprendizaje, recursos empleados y formatos y tecnología empleada. En el campo objetivos, en los casos pertinentes, se han recogido los objetivos señalados de manera explícita. Para la definición de la orientación metodológica se han seguido los modelos educomunicativos propuestos por Kaplún (1998). En las estrategias de enseñanza aprendizaje se ha distinguido si existen propuestas de juego; si se emplea la narración; si se fomenta la resolución de conflictos, la toma de decisiones, la reflexión o la creación de contenidos; si existen espacios para 
Tabla 1. Criterios adaptados a la herramienta de criterios de inclusión y exclusión del método OEPE

\begin{tabular}{|c|c|}
\hline Criterios de inclusión & Criterios de exclusión \\
\hline Condición indispensable: ser una app. & $\begin{array}{l}\text { No ha podido ser descargada o no se ha podido } \\
\text { ejecutar correctamente debido a errores } \\
\text { técnicos. }\end{array}$ \\
\hline $\begin{array}{l}\text { Condición indispensable: aparece un término } \\
\text { del campo semántico de "patrimonio", o, en su } \\
\text { defecto, otro tipo de palabras que pueden estar } \\
\text { relacionadas, como "museo", "tradición" o } \\
\text { "folklore". Si existen dudas con el término } \\
\text { empleado, se tenderá a incluir o, al menos, } \\
\text { documentar para poder localizar en caso } \\
\text { necesario. }\end{array}$ & $\begin{array}{l}\text { Cuenta con una app gemela (contenido e igual } \\
\text { estructura, únicamente cambia el idioma) ya } \\
\text { recogida. }\end{array}$ \\
\hline $\begin{array}{l}\text { Aparece algún término vinculado al ámbito } \\
\text { educativo: enseñanza, didáctica, difusión, } \\
\text { comunicación, sensibilización, concienciación, } \\
\text { etc. }\end{array}$ & $\begin{array}{l}\text { Se trata de una app creada para una acción } \\
\text { concreta de corta duración. }\end{array}$ \\
\hline $\begin{array}{l}\text { Se trata de un diseño educativo: al menos ha de } \\
\text { tener objetivos, si no explícitos, claramente } \\
\text { implícitos o detectables. }\end{array}$ & \multirow{11}{*}{$\begin{array}{l}\text { El contenido presentado no tiene vinculación } \\
\text { alguna con el ámbito cultural. }\end{array}$} \\
\hline $\begin{array}{l}\text { Está impulsado por la administración pública, } \\
\text { museos o centros de interpretación, asociaciones } \\
\text { culturales o empresas de desarrollo tecnológico } \\
\text { en gestión cultural. }\end{array}$ & \\
\hline Se genera desde España. & \\
\hline $\begin{array}{l}\text { Afecta a varias localidades, varias comunidades } \\
\text { e, incluso, varios países. }\end{array}$ & \\
\hline Están implicadas varias entidades. & \\
\hline Se dirige a más de un tipo de público. & \\
\hline $\begin{array}{l}\text { Se dirige, de forma explícita, a públicos con } \\
\text { necesidades educativas específicas. }\end{array}$ & \\
\hline $\begin{array}{l}\text { Se trata de un curso, máster o programa de } \\
\text { formación. }\end{array}$ & \\
\hline Se trata de material didáctico. & \\
\hline $\begin{array}{l}\text { Cuenta con formato } 2.0 \text {, posibilitando la } \\
\text { inclusión de propuestas del usuario. }\end{array}$ & \\
\hline $\begin{array}{l}\text { Ofrece la posibilidad de interacción en términos } \\
\text { de aprendizaje del usuario. }\end{array}$ & \\
\hline
\end{tabular}


compartir, para la creatividad y expresión, para la simulación y experimentación, para la observación, para la indagación y para la investigación. En relación con los recursos empleados, se ha recogido si se presentan videos, textos, mensajes verbales, efectos sonoros, fotografías, mapas, relatos gráficos o dibujos. Por último, en cuanto a los formatos y tecnología empleados, todos los programas inventariados se enmarcan en el campo digital y de tecnología móvil, discriminando aquellos que ofrecen la posibilidad de utilizar Global Positioning Sistem (GPS) o redes sociales (RRSS).

\section{Fase 4: Análisis estadístico descriptivo}

En esta fase se han tenido en cuenta varios datos recogidos en el inventario de programas OEPE, a los que se han incorporado otras variables consideradas importantes para el estudio que nos ocupa.

- Vinculados al apartado dedicado a la identificación y localización, se han considerado los datos recogidos referentes a la entidad responsable o agente, al que, siguiendo la propuesta de Maldonado (2015), se ha sumado una nueva variable que especifica el tipo de agente.

- Del apartado descriptivo se han analizado las variables adaptación a discapacitados y, en caso de que hubiese, tipo de adaptación, también tipo de proyecto -si se trata de un itinerario didáctico o no-. Aunque la ficha OEPE cuenta con un apartado referido al tipo de patrimonio (según las categorías propuestas por la UNESCO), este apartado no se ha rellenado en el proceso de inventariado; sin embargo para el análisis descriptivo sí se ha considerado como una variable, empleando la tipología de patrimonio propuesta por Martín-Cáceres y Cuenca (2015). A estas variables recogidas en la ficha de registro propuesta por OEPE se han incorporado precio y sistema operativo en el que está disponible.

- En cuanto a los datos referidos al diseño educativo, a los objetivos, orientación metodológica, estrategias de enseñanza aprendizaje, recursos empleados y tecnología recogidos en el campo referido al diseño educativo, se han añadido, en el caso de este último campo, las posibilidades de uso de realidad virtual, realidad aumentada y también la posibilidad de curación de contenidos, accesibilidad en la dimensión informacional en función de las posibilidades de adaptación a diferentes niveles y colectivos, la oferta de recursos para profundizar y el rol del enunciatario.

Empleando las variables enumeradas se ha llevado a cabo un análisis de la muestra a través del método estadístico descriptivo, utilizando para ello dos herramientas principales: tablas de frecuencias y cálculos porcentuales, todo ello a través del programa informático SPSS para gestión de los datos y la obtención de resultados.

\section{MUESTRA}

$\mathrm{Al}$ entender que las apps son herramientas de interacción/mediación que tienen una intencionalidad explícita, además de aquellas declaradas educativas, para esta investigación 
se han considerado apps de diferente naturaleza que trabajasen el patrimonio, como por ejemplo guías turísticas. Por lo tanto, contamos con una muestra de carácter determinístico y heterogéneo.

La muestra se ha obtenido mediante la búsqueda y localización de programas descrita en la fase 1 del apartado anterior. Esta exploración, realizada durante los años 2015 y 2016, ha dado lugar a una población de 110 apps de interés para nuestra investigación. A esta se le han aplicado los filtros de discriminación (Fase 2) correspondientes. En esta fase incipiente del proyecto de investigación se ha incorporado a los criterios de exclusión otro que hace referencia a la necesidad de utilizar la app in situ, dado que algunas de ellas requieren del geoposicionamiento para ser utilizadas. Una vez superados todos los filtros designados, se conformó una muestra de 86 apps.

Figura 2. Proceso de filtros y cantidad de apps analizadas

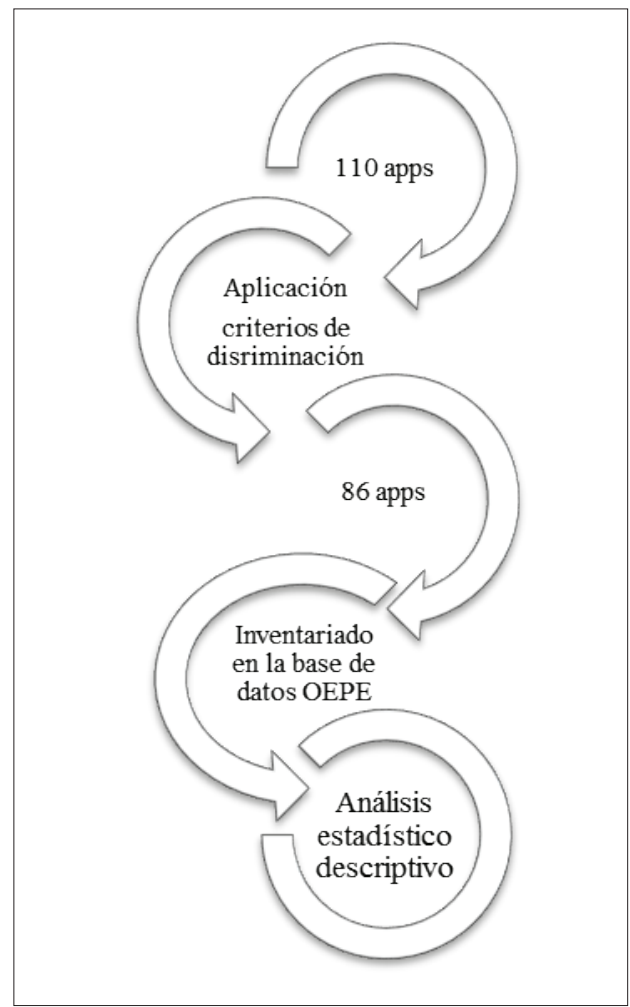

Fuente: elaboración propia. 


\section{RESULTADOS}

Tras seleccionar la muestra, la base de datos nos permite extraer los campos necesarios para iniciar el análisis exploratorio con el objetivo de conocer los programas a través de los tres apartados propuestos por OEPE: identificación y localización, descripción y diseño educativo. Dentro de cada uno de ellos se ha analizado una serie de variables (Tabla 2). En identificación y localización únicamente se ha considerado el tipo de agente que impulsa la app (V1). En el apartado descriptivo se han considerado el precio (V2), el sistema operativo (V3), el tipo de proyecto (V4), la tipología patrimonial (V5), si existe adaptación a públicos con necesidades especiales y el tipo de adaptación (V6) y, por último, la adaptación a diferentes niveles y/o colectivos (V7). En cuanto a la dimensión educativa, las variables analizadas son la existencia o no de objetivos (V8), la orientación metodológica (V9), las estrategias de enseñanza aprendizaje (V10), los recursos empleados (V11), las tecnologías empleadas (realidad aumentada, realidad virtual, geolocalización y redes sociales) (V12), la posibilidad de curación de contenidos (V13), la existencia de recursos para profundizar (V14) y el rol del enunciatario (V15).

Tabla 2. Dimensiones y variables objeto de estudio

\begin{tabular}{|c|c|c|c|}
\hline \multicolumn{2}{|r|}{ Dimensiones } & \multicolumn{2}{|r|}{ Variables } \\
\hline D1 & Identificación y localización & V1 & Agente \\
\hline \multirow[t]{6}{*}{ D2 } & Dimensión descriptiva & V2 & Precio \\
\hline & & V3 & Sistema operativo \\
\hline & & V4 & Tipo de proyecto \\
\hline & & V5 & Tipología patrimonial \\
\hline & & V6 & Adaptación a públicos \\
\hline & & V7 & Adaptación a niveles y/o colectivos \\
\hline \multirow[t]{8}{*}{ D3 } & Dimensión educativa & V8 & Objetivos \\
\hline & & V9 & Orientación metodológica \\
\hline & & V10 & Estrategias de enseñanza aprendizaje \\
\hline & & V11 & Recursos \\
\hline & & V12 & Tecnologías \\
\hline & & V13 & Curación de contenidos \\
\hline & & V14 & Recursos para profundizar \\
\hline & & V15 & Rol del enunciatario \\
\hline
\end{tabular}

Fuente: elaboración propia. 
La Tabla 3, que responde a la V1, muestra que el agente que más apps lanza al mercado es la administración pública, con un 38,4\% (n=33); la institución más implicada son los ayuntamientos. Le siguen los museos públicos y las fundaciones, con el mismo porcentaje $(\mathrm{n}=14 ; 16,3 \%)$. Las apps lanzadas por empresas son $\mathrm{n}=10(11,6 \%)$ y por museos privados o particulares la mitad que las impulsadas por los museos públicos $(n=7 ; 8,1 \%)$. Las universidades y centros de formación son las que menos fomentan el desarrollo de apps en entornos patrimoniales, representando un único caso $(1,2 \%)$.

Tabla 3. Tipo de agente responsable (V1)

\begin{tabular}{|l|c|c|}
\hline Agente & Frecuencia & Porcentaje \\
\hline Administración pública & 33 & $38,4 \%$ \\
\hline Museo/equipamiento cultural público & 14 & $16,3 \%$ \\
\hline Museo/equipamiento cultural privado & 7 & $8,1 \%$ \\
\hline Fundación/asociación & 14 & $16.3 \%$ \\
\hline Marca/empresa & 10 & $11,6 \%$ \\
\hline Universidad/centro de formación & 1 & $1,2 \%$ \\
\hline Persona/proyecto & 7 & $8,1 \%$ \\
\hline Total & 86 & $100 \%$ \\
\hline
\end{tabular}

Fuente: elaboración propia.

En la Tabla 4, respondiendo a la V2 sobre el precio de las apps, se observa que la mayoría de las apps son gratuitas $(n=81 ; 94,2 \%)$, teniendo que pagar más de tres euros solo en un caso $(1,2 \%)$. El resto $(n=4 ; 4,7 \%)$ tienen un coste económico de menos de tres euros. Si bien son gratuitas a la hora de la descarga, algunas apps requieren pagar para acceder a ciertos contenidos o actividades. En este estudio se ha identificado una aplicación de este tipo. En concreto, se trata de un juego en el que el pago permite jugar con otros personajes (Tabla 4).

Tabla 4. Precio (V2)

\begin{tabular}{|l|c|c|}
\hline Precio & Frecuencia & Porcentaje \\
\hline Gratuito & 81 & $94,2 \%$ \\
\hline Menos de 3 euros & 4 & $4,7 \%$ \\
\hline Entre 3 y 10 euros & 1 & $1,2 \%$ \\
\hline Total & 86 & $100 \%$ \\
\hline
\end{tabular}

Fuente: elaboración propia. 
En referencia a los aspectos técnicos (V3), el 74,4\% (n=64) de las aplicaciones son aptas tanto para el sistema operativo de IOS como para el de Android, encontrando solamente en Android el 19,8\% ( $n=17)$ y en IOS el 5,8\% (n=5) (Tabla 5)

Tabla 5. Sistema operativo (V3)

\begin{tabular}{|l|c|c|}
\hline Sistema operativo & Frecuencia & Porcentaje \\
\hline Android & 17 & $19,8 \%$ \\
\hline IOS & 5 & $5,8 \%$ \\
\hline Android e IOS & 64 & $74,4 \%$ \\
\hline Total & 86 & $100 \%$ \\
\hline
\end{tabular}

Fuente: elaboración propia.

De las 86 apps analizadas, los resultados nos indican que el itinerario (V4) es una propuesta muy utilizada $(n=67 ; 77,9 \%)$. Dentro de estos se pueden encontrar autoitinerarios, que son los que ayudan a definir un itinerario basándose en los gustos del usuario y los itinerarios donde el usuario es guiado mediante el recorrido que muestra la app. Puede ser un recorrido o varios a escala local o regional; algunas también ofrecen la posibilidad de crear recorridos a partir de los puntos de interés que el usuario haya seleccionado (Tabla 6).

Tabla 6. Tipo de proyecto (V4)

\begin{tabular}{|l|c|c|}
\hline Tipo de proyecto & Frecuencia & Porcentaje \\
\hline Itinerario & 67 & $77,9 \%$ \\
\hline Otros & 19 & $22,1 \%$ \\
\hline Total & 86 & $100 \%$ \\
\hline
\end{tabular}

Fuente: elaboración propia.

La variable tipología patrimonial (V5) tiene por objetivo conocer qué tipologías patrimoniales son las más trabajadas por las apps. La tipología patrimonial (Tabla 7) que más visibilidad tiene en las apps es la histórica, con un 39,5\% (n=34). La siguiente tipología que más aparece, con un 29,1\% (n=25), es la artística. Un 22,1\% (n=19) de las apps tiene en cuenta el patrimonio en todas sus vertientes y en su globalidad o en más de una faceta, es decir, hace una descripción holística del patrimonio. Finalmente, las tipologías natural y etnológica se pueden considerar residuales al estar representadas solamente en $4(4,7 \%)$ apps. 
Tabla 7. Tipología patrimonial (V5)

\begin{tabular}{|l|c|c|}
\hline Tipología patrimonial & Frecuencia & Porcentaje \\
\hline Etnológico & 4 & $4,7 \%$ \\
\hline Natural & 4 & $4,7 \%$ \\
\hline Histórico & 34 & $39,5 \%$ \\
\hline Artístico & 25 & $29,1 \%$ \\
\hline Holístico & 19 & $22,1 \%$ \\
\hline Total & 86 & $100 \%$ \\
\hline
\end{tabular}

Fuente: elaboración propia.

Por último, dentro de la dimensión descriptiva analizamos las variables sobre adaptación. En primer lugar, presentamos los resultados sobre la adaptación a públicos con necesidades diferentes (V6). Estos resultados (Tabla 8) indican que solo el 23,3\% $(n=20)$ de las aplicaciones están adaptadas a personas con capacidades diferentes, y dentro de estas un 17,4\% ( $\mathrm{n}=15)$ de las aplicaciones permite cambiar el tamaño de la letra, un $12,8 \%(\mathrm{n}=11)$ permite cambiar el tamaño de la pantalla y únicamente un $8,1 \%(\mathrm{n}=7)$ tiene el sistema voiceover.

Tabla 8. Adaptación a personas con capacidades diferentes (V6)

\begin{tabular}{|l|c|c|c|c|c|c|}
\hline \multirow{2}{*}{ Adaptación } & \multicolumn{3}{|c|}{ Frecuencia } & \multicolumn{3}{c|}{ Porcentaje } \\
\cline { 2 - 7 } & Sí & No & Total & Sí & No & Total \\
\hline Personas con capacidades diferentes & 20 & 66 & 86 & $23,3 \%$ & $76,7 \%$ & $100 \%$ \\
\hline Tamaño letra & 15 & 71 & 86 & $17,4 \%$ & $82,6 \%$ & $100 \%$ \\
\hline Cambio pantalla & 11 & 75 & 86 & $12,8 \%$ & $87,2 \%$ & $100 \%$ \\
\hline Voiceover & 7 & 79 & 86 & $8,1 \%$ & $91,9 \%$ & $100 \%$ \\
\hline
\end{tabular}

Fuente: elaboración propia.

En cuanto a la adaptación a diferentes niveles y colectivos (V7), los datos (Tabla 9) indican que en la mayoría de los casos la información no está adaptada a diferentes colectivos, como pueden ser los niños y niñas $(n=4 ; 4,7 \%)$, ni se plantean diferentes niveles dentro de la aplicación $(\mathrm{n}=11 ; 12,8 \%)$. 
Tabla 9. Adaptación a diferentes niveles y colectivos (V7)

\begin{tabular}{|l|c|c|c|c|c|c|}
\hline \multirow{2}{*}{ Adaptación } & \multicolumn{3}{|c|}{ Frecuencia } & \multicolumn{3}{c|}{ Porcentaje } \\
\cline { 2 - 7 } & Sí & No & Total & Sí & No & Total \\
\hline Diferentes niveles & 11 & 75 & 86 & $12,8 \%$ & $87,2 \%$ & $100 \%$ \\
\hline Diferentes colectivos & 4 & 82 & 86 & $4,7 \%$ & $95,3 \%$ & $100 \%$ \\
\hline
\end{tabular}

Fuente: elaboración propia.

Dentro de la dimensión educativa la tabla 10 nos informa de que la mayoría de las apps no especifican de manera explícita sus objetivos educativos (V8) $(n=70 ; 81,4 \%)$.

Tabla 10. Objetivos educativos (V8)

\begin{tabular}{|l|c|c|}
\hline Objetivos & Frecuencia & Porcentaje \\
\hline Sí & 16 & $18,6 \%$ \\
\hline No & 70 & $81,4 \%$ \\
\hline Total & 86 & $100 \%$ \\
\hline
\end{tabular}

Fuente: elaboración propia.

Los resultados de la Tabla 11 señalan que la orientación metodológica (V9) más utilizada en las apps es la que pone énfasis en los contenidos $(n=52 ; 60,5 \%)$, seguida de la que pone énfasis en los resultados $(n=32 ; 37,2 \%)$, y la menos utilizada corresponde a la que pone el acento en el proceso $(n=2 ; 2,3 \%)$.

Tabla 11. Orientación metodológica (V9)

\begin{tabular}{|l|c|c|}
\hline Modelo educomunicativo & Frecuencia & Porcentaje \\
\hline Énfasis en los contenidos & 52 & $60,5 \%$ \\
\hline Énfasis en los resultados & 32 & $37,2 \%$ \\
\hline Énfasis en el proceso & 2 & $2,3 \%$ \\
\hline Total & 86 & $100 \%$ \\
\hline
\end{tabular}

Fuente: elaboración propia.

En cuanto a la variable que hace referencia a las estrategias de enseñanza aprendizaje (V10) los resultados (Tabla 12) indican que un 61,6\% $(n=53)$ de las apps ofrecen espacios 
para la observación mientras que el resto de las estrategias de enseñanza aprendizaje se utilizan mínimamente. Las siguientes estrategias más utilizadas son el espacio para la indagación con un 30,2\% ( $n=26)$, el fomento de la reflexión con un 19,8\% ( $n=17)$, el juego con $18,6 \%(\mathrm{n}=16)$, los espacios para la simulación y experimentación con un $12,8 \%(\mathrm{n}=11)$ y la toma con de decisiones con un 10,5\% (9). El resto no supera el 10\%: creación de contenidos $(n=8 ; 9,3 \%)$, espacios para la creatividad y expresión $(n=8 ; 9,3 \%)$, espacios para compartir $(n=8 ; 9,3 \%)$, narraciones $(n=7 ; 8,1 \%)$, espacios para la investigación $(n=5$; $5,8 \%)$ y la menos utilizada, la resolución de conflictos con una sola app $(1,2 \%)$.

Tabla 12. Estrategias de enseñanza aprendizaje (V10)

\begin{tabular}{|l|c|c|c|c|c|c|}
\hline \multirow{2}{*}{ Estrategias de enseñanza aprendizaje } & \multicolumn{3}{|c|}{ Frecuencia } & \multicolumn{3}{c|}{ Porcentaje } \\
\cline { 2 - 7 } & Sí & No & Total & Sí & No & Total \\
\hline Toma de decisiones & 9 & 77 & 86 & $10,5 \%$ & $89,5 \%$ & $100 \%$ \\
\hline Fomento de la reflexión & 17 & 69 & 86 & $19,8 \%$ & $80,2 \%$ & $100 \%$ \\
\hline Creación de contenidos & 8 & 78 & 86 & $9,3 \%$ & $90,7 \%$ & $100 \%$ \\
\hline Espacios para compartir & 8 & 78 & 86 & $9,3 \%$ & $90,7 \%$ & $100 \%$ \\
\hline Espacios para la creatividad y expresión & 8 & 78 & 86 & $9,3 \%$ & $90,7 \%$ & $100 \%$ \\
\hline Espacios para la simulación y experimentación & 11 & 75 & 86 & $12,8 \%$ & $87,2 \%$ & $100 \%$ \\
\hline Espacios para la observación & 53 & 33 & 86 & $61,6 \%$ & $38,4 \%$ & $100 \%$ \\
\hline Espacios para la indagación & 26 & 60 & 86 & $30,2 \%$ & $69,8 \%$ & $100 \%$ \\
\hline Espacios para la investigación & 5 & 81 & 86 & $5,8 \%$ & $94,2 \%$ & $100 \%$ \\
\hline Juego & 16 & 70 & 86 & $18,6 \%$ & $81,4 \%$ & $100 \%$ \\
\hline Narraciones & 7 & 79 & 86 & $8,1 \%$ & $91,9 \%$ & $100 \%$ \\
\hline Resolución de conflictos & 1 & 85 & 86 & $1,2 \%$ & $98,8 \%$ & $100 \%$ \\
\hline
\end{tabular}

Fuente: elaboración propia.

En la Tabla 13 vemos que el recurso educativo (V11) más utilizado en las aplicaciones es el texto $(n=79 ; 91,9 \%)$, seguido de las fotografías $(n=72 ; 83,7 \%)$ y de los mapas $(n=66$; $76,7 \%$ ). Del resto de recursos educativos se utilizan más de un $25 \%$ los mensajes verbales $(n=31 ; 36 \%)$, los efectos sonoros $(n=26 ; 30,2 \%)$ y los dibujos $(n=23 ; 26,7 \%)$. Finalmente, los tipos de recursos educativos menos frecuentes son los videos ( $\mathrm{n}=17 ; 19,8 \%)$, y el menos utilizado, los relatos gráficos $(n=2 ; 2,3 \%)$. De esta manera, podemos comprobar que los recursos educativos son incluyentes, es decir, que la mayoría de las apps utilizan diversos recursos educativos para ser más eficaces en la recepción del mensaje. 
Estudios Pedagógicos XLIII, N 4: 115-136, 2017

CARACTERÍSTICAS Y DIMENSIÓN EDUCATIVA EN APPS DE EDUCACIÓN PATRIMONIAL. ANÁLISIS A PARTIR DEL MÉTODO OEPE

Tabla 13. Recursos educativos (V11)

\begin{tabular}{|l|c|c|c|c|c|c|}
\hline \multirow{2}{*}{ Recursos educativos } & \multicolumn{3}{|c|}{ Frecuencia } & \multicolumn{3}{c|}{ Porcentaje } \\
\cline { 2 - 7 } & Sí & No & Total & Sí & No & Total \\
\hline Video & 17 & 69 & 86 & $19,8 \%$ & $80,2 \%$ & $100 \%$ \\
\hline Textos & 79 & 7 & 86 & $91,9 \%$ & $8,1 \%$ & $100 \%$ \\
\hline Mensajes verbales & 31 & 55 & 86 & $36 \%$ & $64 \%$ & $100 \%$ \\
\hline Efectos sonoros & 26 & 60 & 86 & $30,2 \%$ & $69,8 \%$ & $100 \%$ \\
\hline Fotografías & 72 & 14 & 86 & $83,7 \%$ & $16,3 \%$ & $100 \%$ \\
\hline Mapas & 66 & 20 & 86 & $76,7 \%$ & $23,3 \%$ & $100 \%$ \\
\hline Relatos gráficos & 2 & 84 & 86 & $2,3 \%$ & $97,7 \%$ & $100 \%$ \\
\hline Dibujos & 23 & 63 & 86 & $26,7 \%$ & $73,3 \%$ & $100 \%$ \\
\hline
\end{tabular}

Fuente: elaboración propia.

Otra variable investigada es la utilización de tecnologías que permiten crear experiencias inmersivas como la realidad virtual, la realidad aumentada y la geolocalización; y la utilización de las redes sociales para compartir información (V12). La realidad aumentada es la visualización de información mezclada con objetos reales y la utilizan 11 apps $(12,8 \%)$. La realidad virtual hace referencia a la recreación de un entorno de apariencia real y la encontramos en 8 apps $(9,3 \%)$. La geolocalización permite situar un objeto en una ubicación determinada, esta herramienta la presentan algo más de la mitad de las apps investigadas $(\mathrm{n}=44 ; 51,2 \%)$. Por último, las redes sociales nos permiten compartir la información y menos de la mitad $(n=31 ; 36 \%)$ de las apps dan esta posibilidad (Tabla 14).

Tabla 14. Tecnologías que crean experiencias inmersivas (V12)

\begin{tabular}{|l|c|c|c|c|c|c|}
\hline \multirow{2}{*}{$\begin{array}{l}\text { Tecnologías que crean } \\
\text { experiencias inmersivas }\end{array}$} & \multicolumn{3}{|c|}{ Frecuencia } & \multicolumn{3}{c|}{ Porcentaje } \\
\cline { 2 - 7 } & Sí & No & Total & Sí & No & Total \\
\hline Realidad aumentada & 11 & 75 & 86 & $12,8 \%$ & $87,2 \%$ & $100 \%$ \\
\hline Realidad virtual & 8 & 78 & 86 & $9,3 \%$ & $90,7 \%$ & $100 \%$ \\
\hline Geolocalización & 44 & 42 & 86 & $51,2 \%$ & $48,8 \%$ & $100 \%$ \\
\hline Redes sociales & 31 & 55 & 86 & $36 \%$ & $64 \%$ & $100 \%$ \\
\hline
\end{tabular}

Fuente: elaboración propia.

En la Tabla 15 podemos ver que la curación de contenidos o la posibilidad de crear contenidos (V13) por parte de las personas usuarias de las apps es mínima, ya que únicamente $11(12,8 \%)$ lo permiten. 
Tabla 15. Curación de contenidos (V13)

\begin{tabular}{|l|c|c|}
\hline Curación de contenidos & Frecuencia & Porcentaje \\
\hline Sí & 11 & $12,8 \%$ \\
\hline No & 75 & $87,2 \%$ \\
\hline Total & 86 & $100 \%$ \\
\hline
\end{tabular}

Fuente: elaboración propia.

Relacionado con el campo del diseño educativo, la variable sobre los recursos para profundizar en la temática e información de las apps (V14) nos indica (Tabla 16) que el $72,1 \%(n=62)$ de las aplicaciones no está dotada de recursos que ayuden al usuario a profundizar en los conceptos e informaciones que quiere transmitir la app.

Tabla 16. Recursos para profundizar (V14)

\begin{tabular}{|l|c|c|}
\hline Recursos para profundizar & Frecuencia & Porcentaje \\
\hline Sí & 24 & $27,9 \%$ \\
\hline No & 62 & $72,1 \%$ \\
\hline Total & 86 & $100 \%$ \\
\hline
\end{tabular}

Fuente: elaboración propia.

Por último, la variable sobre el rol del enunciatario (V15), es decir, sobre quién recoge la información, permite comprobar que el $68,8 \%(n=59)$ de las apps se dirigen al usuario de manera anónima, mientras que solo un $24,4 \%(n=21)$ tiene en consideración la persona a la que se dirige (Tabla 17).

Tabla 17. Rol del enunciatario (V15)

\begin{tabular}{|l|c|c|}
\hline Rol del enunciatario & Frecuencia & Porcentaje \\
\hline Está presente & 21 & $24,4 \%$ \\
\hline Anónimo & 59 & $68,6 \%$ \\
\hline Destinatario específico & 6 & $7 \%$ \\
\hline Total & 86 & $100 \%$ \\
\hline
\end{tabular}

Fuente: elaboración propia. 


\section{CONCLUSIONES}

La primera conclusión a la que llegamos tras analizar los datos en relación con las apps de educación patrimonial es que la oferta de este tipo de app, de contenido patrimonial, se encuentra en constante expansión en los contextos de enseñanza aprendizaje informal. En los espacios patrimoniales las apps se han convertido en un recurso cada vez más dinámico y flexible, favoreciendo una experiencia educativa más cercana, vivencial y accesible, posibilitando al usuario el acceso a contenidos interactivos, así como enriqueciendo la experiencia en torno a un determinado bien patrimonial, hablamos, por tanto, de una hipertextualidad con una geometría humana y creativa (Falcón \& Torregrosa, 2014).

Aún así, cuando nuestro análisis se centra en la dimensión educativa comprobamos que, incluso entendiendo las apps como un recurso didáctico, los agentes que las impulsan no cuidan del todo esta faceta, descuidando en muchos casos el diseño didáctico de las mismas y olvidando que la utilización de la tecnología y la hipertextualidad unida a esta no es educativa en sí misma, y necesita tanto de un tratamiento didáctico como de hiperlectores formados (Burbules \& Casllister, 2008).

Esto lo comprobamos en la falta de objetivos que presenta la mayoría de las apps analizadas $(18,65 \%)$, cuya presencia consideramos imprescindible para un buen diseño de herramientas didácticas. Si tenemos en cuenta la metodología y las estrategias de enseñanza aprendizaje utilizadas por las apps deducimos que, a pesar de la diversidad de estrategias empleadas, como pueden ser la observación y reflexión, simulación y experimentación, o creación de contenidos, las más empleadas siguen siendo las de corte más tradicional, centradas en la experiencia visual a través de la lectura y las imágenes, en las que la información se muestra de manera unidireccional. Este tipo de estrategias de enseñanza aprendizaje se encuadran dentro de un modelo metodológico que pone el énfasis en los contenidos (60,5\%). Es decir, parte de la idea de que es el conocimiento experto el que forma a los educandos, quienes adquieren un rol pasivo en el proceso de enseñanza aprendizaje. De esta manera, se da mayor importancia a los conceptos transmitidos, en perjuicio de procesos procedimentales o de cuestiones más actitudinales.

Así, los resultados de esta investigación son acordes con los de la investigación de Grevtova (2016) sobre la escasa utilización del modelo colaborativo en las apps de patrimonio urbano, ya que únicamente dos $(2,3 \%)$ de las apps analizadas ponen énfasis en el proceso, que se basa en el diálogo y la reflexión, exigiendo la máxima participación de las personas usuarias. Aun así, no son pocas las apps que ponen el énfasis en los resultados (37,2\%), dando la oportunidad de que la persona usuaria participe contestando a preguntas o superando retos.

A tenor de todo lo expuesto, podemos señalar que, por lo general, las apps de educación patrimonial desarrolladas hasta el año 2016 en España infrautilizan sus posibilidades educativas, ya que no ofrecen espacios en los que las personas usuarias puedan generar contenidos $(12,8 \%)$ con los que se identifiquen. Esta opción posibilita que se enriquezcan las relaciones de estas personas con el patrimonio ofertado desde la app, pasando a ser un patrimonio vivido y no solo observado, y en el mejor de los casos, puede ofrecer la oportunidad de participar en los procesos de patrimonialización. La generación de contenidos, la toma de decisiones y el enriquecimiento de las relaciones con el patrimonio de forma personalizada, interactiva e involucrando el aspecto emocional debieran favorecer la transición de una utilización pasiva de la app hacia la consideración de usuarios y usuarias implicadas en sus propios procesos de enseñanza aprendizaje. El fomento del uso de redes 
sociales desde las mismas apps puede favorecer estas cuestiones, resultando especialmente interesante entre las generaciones más jóvenes, más tendientes al uso de las mismas y futuro público al que hay que educar en la valoración y defensa del patrimonio. Somos de la opinión de que su sola inclusión conseguiría aumentar la interacción entre las personas usuarias, y entre estas y el bien patrimonial. En definitiva, se lograría una mejor experiencia y mayor implicación de las personas consiguiendo, como señala Fontal (2003), que el protagonismo del proceso de patrimonialización pase del objeto al sujeto que es quien, al comprenderlo, respetarlo, valorarlo, cuidarlo y disfrutarlo llegará, en última instancia, a transmitirlo, secuencia base del conocimiento. Por lo tanto, nos parece imprescindible la incorporación de las redes sociales en las apps de corte patrimonial, más aún si tenemos en cuenta su sencilla utilización.

Por otro lado, los resultados nos indican que se infrautilizan las posibilidades que los dispositivo móviles tienen para adaptarse a diferentes niveles $(12,8 \%)$, colectivos $(4,7 \%)$ o personas con capacidades diferentes $(23,3 \%)$. En la mayoría de las apps analizadas el rol del enunciatario es anónimo, por lo que el discurso es uniforme, sin adaptar y, generalmente, sin apelar al lado emotivo del discurso. El 24,4\% de las veces en las que el enunciatario está presente, se busca una interacción con el usuario o usuaria, algo que debiera aumentar si se desea que fidelice con la app, llegue a hacer suyo el patrimonio y desarrolle un aprendizaje significativo.

A través de los datos obtenidos, esta investigación nos ayuda a extraer aquellas claves que permiten profundizar en el estado de la cuestión en el campo de las apps de carácter patrimonial desarrolladas en España. En este sentido, cabe destacar la implicación de las administraciones públicas en el desarrollo de este tipo de apps, así como la gratuidad de las mismas, o su diseño tanto para sistemas IOS como para Android en la mayoría de los casos analizados. Este dato tiene especial relevancia, pues indica la búsqueda de un mayor alcance y un mayor público potencial. Permite subrayar el carácter social y cultural de la educación patrimonial, así como la libertad y gratuidad en el acceso a recursos educativos cuyos contenidos se vinculan a bienes compartidos, tratando así de satisfacer una necesidad social fundamental a través de las tecnologías móviles. Esto, unido a la popularidad de los smartphones y tablets como herramientas de información, comunicación y almacenamiento de datos, favorece su uso como instrumento de aprendizaje.

En conclusión, lo dicho hasta ahora nos ayuda a contextualizar el futuro de las apps como herramientas de apoyo para el enriquecimiento de una experiencia vivencial, real, compartida y situada en un determinado espacio de patrimonio, ante un determinado bien patrimonial, siempre que se utilicen metodologías que se centren en las personas usuarias, es decir, les permitan generar contenidos, tomar decisiones y enriquecer las relaciones con el patrimonio de una forma personalizada, interactiva e involucrando el aspecto emocional, lo que llamaríamos una hipertextualidad creativa, basada en espacio de creación y divagación, con una clara geometría humana. Pensamos que estas pueden ser algunas de las claves para favorecer la transición de las apps informativas o transmisivas, más numerosas al día de hoy, hacia unas apps más educativas y acordes a lo que marca la educación patrimonial. Es decir, el análisis de la muestra nos permite esbozar las líneas de continuidad deseables a favor de procesos de enseñanza aprendizaje de mayor calidad, bidireccionales, activos, que permitan una mayor implicación de las personas usuarias y la creación de contenidos que puedan ser compartidos en red, enriqueciendo la dimensión relacional e identitaria que se sitúa en la base de todo bien o espacio patrimonial. 
Siguiendo el método OEPE, nuestros próximos estudios tienen como fin identificar aquellos programas o aplicaciones que cumplan unos estándares de calidad que los identifique como referentes para, posteriormente, evaluarlos en mayor profundidad. Así, a través de su conocimiento, podremos ir definiendo el modelo de apps de educación patrimonial.

\section{REFERENCIAS BIBLIOGRÁFICAS}

Brazuelo, F., \& Gallego, D. J. (2011). Mobile learning: Los dispositivos móviles como recurso educativo. Sevilla: Eduforma.

Burbules, N. (2012). El aprendizaje ubicuo y el futuro de la enseñanza. Encounters/Encuentros/ Rencontres on Education, 13, 3-14.

Burbules, N., \& Callister, T. (2008). Educación: Riesgos y promesas de las nuevas tecnologías de la información. Buenos Aires: Granica.

Cabero, J., \& López Meneses, E. (2009). Descripción de un instrumento didáctico para el análisis de modelos y estrategias de enseñanza de cursos universitarios en red (A.D.E.C.U.R.). Pixel-Bit. Revista de Medios y Educación, 34, 13-30.

Crescenzi, L., \& Grané, M. (2016) Análisis del diseño interactivo de las mejores APPs educativas para niños de cero a ocho años. Comunicar: Revista Científica Iberoamericana de Comunicación y Educación, 46, 77-85.

Cuenca, J.M. (2002). El patrimonio en la didáctica de las ciencias sociales: análisis de concepciones, dificultades y obstáculos para su integración en la enseñanza obligatoria (Tesis inédita de doctorado). Universidad de Huelva.

Cuenca, J.M., \& Estepa, J. (2013). La educación patrimonial: líneas de investigación actual y nuevas perspectivas. En J. Estepa (Ed.), La educación patrimonial en la escuela y el museo: investigación y experiencias (pp. 343-356). Huelva: Universidad de Huelva.

Economou, M., \& Meintani, E. (2011). Promising beginnings? Evaluating museum mobile phone $A P P s$. Artículo presentado en el congreso Re-thinking technology in museums. Emerging Experiences, University of Limerick, Ireland. Recuperado de www.idc.ul.ie/techmuseums11/ paper/paper8.pdf /

Falcón, R.M., \& Torregrosa, A. (2014). Ser el sitio. Recuperado de https://www.academia. edu/12978771/Ser_el_sitio. Recuperado el 19 de Julio de 2017.

Fontal, O. (2003). La educación patrimonial. Teoría y práctica para el aula, el museo e internet. Gijón: Trea.

Fontal, O. (2013). El patrimonio en la escuela: más allá del patrimonio como contenido curricular. En O. Fontal (Coord.), La educación patrimonial. Del patrimonio a las personas (pp. 23-44). Gijón: Trea.

Fontal, O. (2016). The Spanish Heritage Education Observatory. Cultura y Educación, 28(1), 254266. doi:10.1080/11356405.2015.1110374.

Gómez-Redondo, C. (2013). Procesos de Patrimonialización en el arte contemporánea: diseño de un artefacto educativo para la identización (Tesis inédita de doctorado). Universidad de Valladolid.

Grevtsova, I. (2016). Tendencias del uso de las tecnologías móviles en espacios urbanos: m-learning y patrimonio cultural. Revista $P H, 90,132-151$.

Guerrero, C. (2015). UMUMOOC Una propuesta de indicadores de calidad pedagógica para la realización de cursos MOOC. Campus Virtuales, 4(2), 70-76.

Ibáñez-Etxeberria, A., \& Kortabitarte, A. (2016). Apps, redes sociales y dispositivos móviles en educación patrimonial. IPCE. http://www.mecd.gob.es/planes-nacionales/dam/jcr:4cc8d5a23084-409e-a134-b46b62f7126e/apps-y-socialmedia-en-educacionpatrimonial.pdf 
Ibáñez-Etxeberria, A., Fontal, O., Vicent, N., \& Gillate, I. (2014). Educación y patrimonio: Análisis de la oferta educativa de museos para la formación de la ciudadanía en la etapa 0-6 en España. Museologia e Patrimônio, 7(2), 3-14.

Imbert-Bouchard D., Llonch N., Martín Piñol, C., \& Osácar, E. (2013). Turismo cultural y APPs. Un breve panorama de la situación actual. HeryMus. Heritage y Museography, 13. Recuperado de http://www.trea.es/books/hermus-heritage-museography-13

Instituto Nacional de Estadística (INE). (2016). Encuesta sobre Equipamiento y Uso de Tecnologías de Información y Comunicación en los hogares. Autor. Recuperado de http://www.ine.es/prensa/ tich_prensa.htm

Kaplún, M. (1998). Una pedagogía de la comunicación. Madrid: Ediciones de la Torre.

Kukulska-Hulme, A. (2007). Mobile Usability in Educational Contexts: What have we learnt? The International Review of Research in Open and Distributed Learning, 8(2), 1-16. Recuperado el 8 de enero del 2016 desde http://www.irrodl.org/index.php/irrodl/rt/printerFriendly/356/879

Lee, C-Y., \& Cherner, T. S. (2015). A comprehensive evaluation rubric for assessing instructional APPs. Journal of Information Technology Education: Research, 14, 21-53.

López Benito, M. (2014). Estudio exploratorio sobre la interpretación didáctica del arte en el museo a través de tecnologías móviles (Tesis Doctoral). Universitat de Barcelona.

López Benito, M., Martínez Gil, T., \& Santacana, J. (2014). Aplicaciones: ¿también instrumentos educativos para descodificar el mundo de los museos y la cultura? En J. Santacana \& M. López Benito (Coords.), Educación, tecnología digital y patrimonio cultural. Para una educación inclusiva (pp. 71-84). Gijón: Ediciones Trea.

Maldonado, M.S. (2015). Educación Patrimonial y redes sociales. Análisis y evaluación de acciones en los medios de comunicación social para la definición de una cartografía educativa (Tesis inédita de doctorado). Universidad de Valladolid.

Marín-Cepeda, S. (2014). Educación patrimonial y diversidad: evaluación de programas y definición de un modelo basado en los procesos de patrimonialización (Tesis inédita de doctorado). Universidad de Valladolid.

Marín-Cepeda, S., García-Ceballos, S., Vicent, N., Gillate, I., \& Gómez-Redondo, C. (2017). Educación Patrimonial Inclusiva en OEPE: un estudio prospectivo. Revista de Educación, 375, 110-135. doi:10.4438/1988-592X-RE-2016-375-337

Martín Cáceres, M., \& Cuenca López, J. M. (2015). Educomunicación del patrimonio. Educatio Siglo XXI, 33(1) Recuperado el 15 de abril de 2017 desde http://revistas.um.es/educatio/article/ view/222491

Martín-Cáceres, M., \& Cuenca, J. M. (2015). Educomunicación del patrimonio. Educatio Siglo XXI, 33(1), 33-54.

Mazanec, J. A., Crotts, J. C., Gursoy, D., \& Lu, L. (2015). Homogeneity versus heterogeneity of cultural values: An item-response theoretical approach applying Hofstede's cultural dimensions in a single nation. Tourism Management, 48(1), 299-304.

Osuna, S. (2011). Aprender en la Web 2.0. Aprendizaje colaborativo en comunidades virtuales. $\mathrm{La}$ Educ@cion. Revista Digital, 45, 1-19.

Rodríguez, J. (2001) Métodos de muestreo. Madrid: Centro de Investigaciones Sicológicas.

Sampson, D.G. \& Zervas, P. (2013). Context-aware adaptative and personalized Mobile Learning Systems. In D.G. Sampson, D. Ifenthaler \& M. Spector (Eds.) Ubiquitous and Mobile Learning in the Digital Age (pp. 3-17). New York: Springer.

Sánchez, A. (2016). Memoria, identidad y comunidad: evaluación de programas de educación patrimonial en la comunidad de Madrid (Tesis inédita de doctorado). Universidad de Valladolid.

Sharples, M., Meek, J., Vavoula, G., Lonsdale, P., \& Rudman, P. (2007). An Evaluation of MyArtSpace: a Mobile Learning Service for School Museum Trips. Proceedings of mLearn 2007 Conference, Melbourne, Australia.

Stake, R. E. (2004). Standards-based \& responsive evaluation. Thousand Oaks: Sage Publications. 
Tallon, L. (2013). Mobile Strategy in 2013: an analysis of the annual. Museums y Mobile survey. Pocket-Proof. Recuperado de http://www.museums-mobile.org/survey.

UNESCO (2013). Enfoques estratégicos sobre las TICs en educación en América Latina y el Caribe. Recuperado de http://www.unesco.org/new/fileadmin/MULTIMEDIA/FIELD/Santiago/images/ ticsesp.pdf

Vavoula, G. (2005). Report on literature on mobile learning, science and collaborative acitivit (No. D33.2.2, Final). Kaleidoscope. Recuperado de http://halshs.archives-ouvertes.fr/ docs/00/19/01/75/PDF/Vavoula-Kaleidoscope-2005.pdf.

Villalonga, C., \& Marta-Lazo, C. (2015). Modelo de integración educomunicativa de "APPs" móviles para la enseñanza y aprendizaje. Píxel-Bit. Revista De Medios y Educación, 46, 137153. doi:10.12795/pixelbit.2015.i46.09

Vincent, T. (2012). Ways to Evaluate Educational APPs [Blog]. Learning in hand with Tony Vincent. Recuperado desde http://learninginhand.com/blog/ways-to-evaluate-educational-APPs.html3 